\title{
Identifying Key Transmission Route of Avian Influenza A(H9N2) in Live Poultry Markets
}

\author{
Eric H. Lau*, Shengqiu Zhang, Connie Leung, Benjamin J. Cowling, Joseph T. Wu and \\ Malik Peiris
}

School of Public Health, The University of Hong Kong, Hong Kong, Hong Kong

\section{Objective}

This study assessed the transmission of low pathogenic avian influenza in live poultry market setting, using paired fecal and drinking water samples from a longitudinal surveillance program. The relative contribution of transmission via direct fecal-oral route versus drinking water will be determined.

\section{Introduction}

Live poultry markets (LPMs) continue to operate in many Asian countries. Low pathogenic avian influenza (LPAI) viruses are often endemic in the poultry, and LPM presents the opportunity for human-poultry interactions and potential human infections with avian influenza viruses.

As a series of interventions to control avian influenza transmission in Hong Kong LPMs, local health authority implemented market rest days once every month since mid-2001, and an additional rest day every month since 2003 , during which all unsold poultry were slaughtered and the stalls cleaned and disinfected. Rest days were found to effectively reduce avian influenza $\mathrm{A}(\mathrm{H} 9 \mathrm{~N} 2)$ isolation rate to baseline level for a few days following the rest days. However, H9N2 isolation rate was still observed to be increasing between the rest days, indicating the existence of efficient transmission in spite of rapid turnover of poultry.

In LPMs, poultry are usually stored in cages where drinking water is shared among poultry. This is analogous to environmental contamination in the wild, but transmissibility may even be higher due to the dense environment. The use of drinking water for avian influenza surveillance in LPM setting was suggested to be more sensitive than fecal samples (1). However, the relative contribution of direct fecal-oral versus water transmission routes in the LPM setting was not yet understood. This study aimed to determine their role, which will have implications in the control of avian influenza transmission.

\section{Methods}

We analyzed 7,321 paired fecal and drinking water samples from a longitudinal surveillance programme during the period with 2 monthly rest days in the LPMs. Samples were collected from chicken cages and subsequently cultured. Positive isolates were subtyped by hemagglutination-inhibition tests and neuraminidase inhibition test. Data were aggregated by sampling occasion and days after the rest days.

A compartmental transmission model which incorporated turnover and overnight stay of poultry, virus contamination and decay in drinking water was fitted to the data (Figure 1). A 12-hour trading day was assumed. Based on the parameterized model, we simulated the scenario that water transmission was prohibited to assess the role of transmission via drinking water.

\section{Results}

H9N2 isolation rates ranged from $0-25 \%$ for fecal samples and $0-56 \%$ for drinking water samples. A clear increasing trend can be seen over days after the rest days (Figure 2). The estimated parameter for water transmission is higher than the parameter for direct fecal-oral transmission. Simulation results show that transmission via drinking water plays a major role in the amplification of LPAI in the LPM setting (Figure 2).

\section{Conclusions}

Our study showed that drinking water has a major role in the transmission and amplification of LPAI H9N2 in LPMs, comparing to direct fecal-oral transmission route. Given the relatively low prevalence of H9N2 in chicken, direct transmission is governed by chance events, while chickens are consistently exposed to viruses in drinking water if contaminated. Drinking water could be targeted for intervention to control LPAI transmission in LPM. The use of drinking fountain or frequent disinfection of drinking water may be considered. Avian influenza viruses (e.g. H5N1) may differ in their pattern of virus shedding via oral versus fecal routes and thus extrapolation of these results to other viruses needs to be done with caution. However, H7N9 viruses are similar to H9N2 viruses by being shed primarily via the respiratory / oral route (2) and it is reasonable to assume that these conclusions would apply to H7N9 virus which is of major public health concern. However, our model could not differentiate the effect of indirect fecal-oral transmission through contamination of drinking water by droppings versus contamination through drinking.

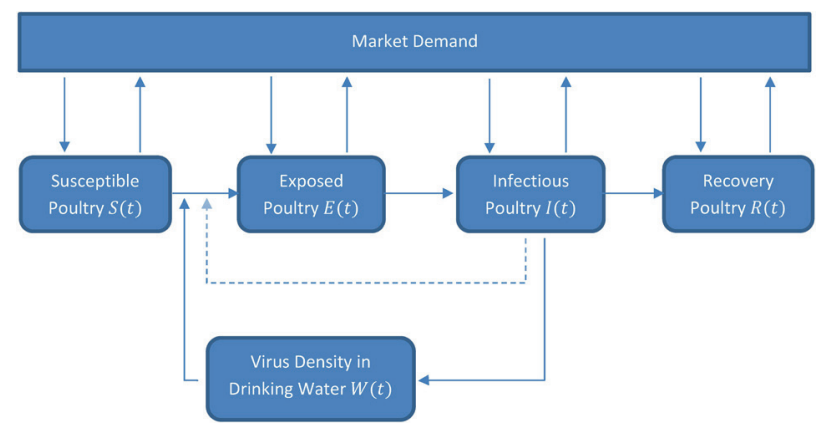

Framework of the compartmental transmission model 

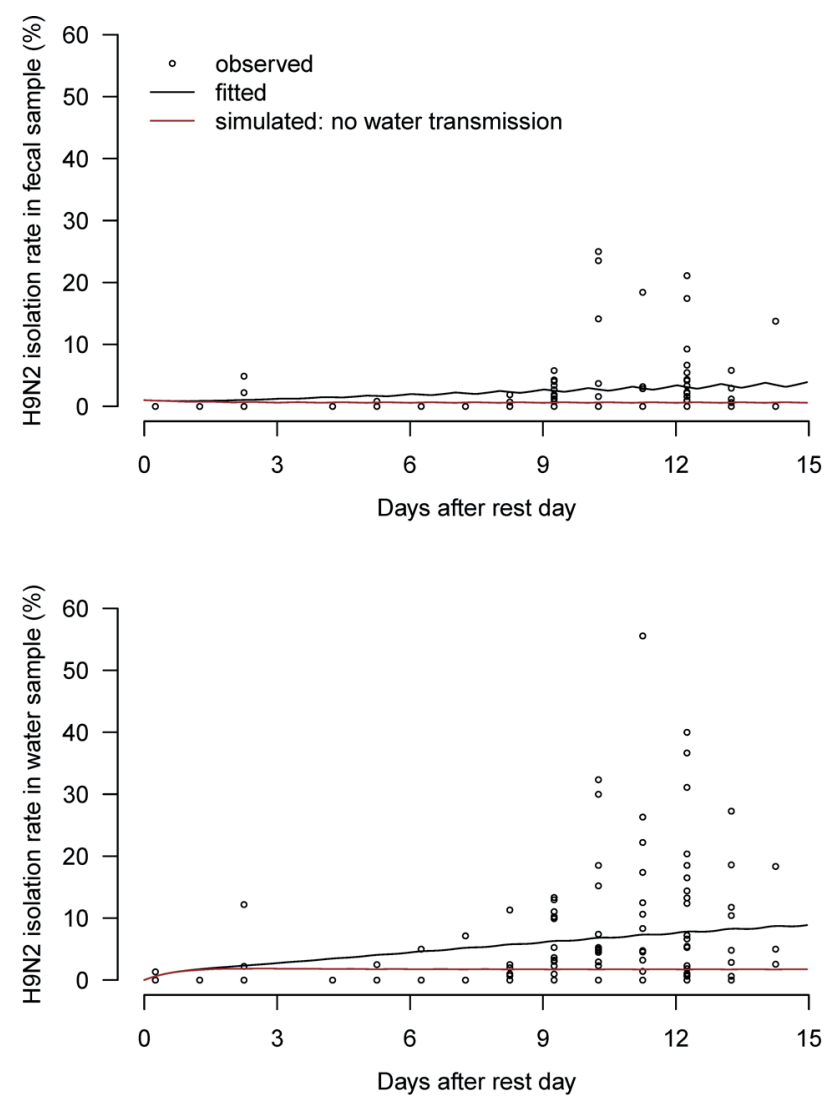

Influenza A(H9N2) isolation rates from fecal and water samples

\section{Keywords}

Avian influenza; influenza A(H9N2); live poultry market; fecal-oral; drinking water

\section{Acknowledgments}

This work is supported by the Harvard Center for Communicable Disease Dynamics from the National Institute of General Medical Sciences, the Area of Excellence Scheme of the Hong Kong University Grants Committee, and the Health and Medical Research Fund of the Food and Health Bureau, Government of the Hong Kong Special Administrative Region.

\section{References}

1. Leung YHC et al. Poultry drinking water used for avian influenza surveillance. Emerg Infect Dis. 2007;13(9):1380-2.

2. Luk GS et al. Transmission of H7N9 Influenza Viruses with a Polymorphism at PB2 Residue 627 in Chickens and Ferrets. $J$ Virol. 2015 Oct;89(19):9939-51.

\section{*Eric H. Lau}

E-mail: ehylau@hku.hk 